“C 2010 IEEE. Personal use of this material is permitted. Permission from IEEE must be obtained for all other uses, in any current or future media, including reprinting/republishing this material for advertising or promotional purposes, creating new collective works, for resale or redistribution to servers or lists, or reuse of any copyrighted component of this work in other works.” 


\title{
A User Level Markov Model for Service Based CRRM Algorithm
}

\author{
Leijia Wu, Abdallah E. AL Sabbagh, Kumbesan Sandrasegaran, and Maged Elkashlan \\ University of Technology, Sydney and CSIRO ICT Centre, Australia \\ E-mail: \{lewu,aSabbagh, kumbes\}@eng.uts.edu.au, maged.elkashlan@csiro.au
}

\begin{abstract}
In order to support the conceptual development of Radio Access Technology (RAT) selection algorithms, the theory of Markov model has been used. Performance metrics can be derived from the steady state probabilities of a Markov model. This paper extends a User Level Markov model for a three colocated RATs system from existing two co-located RATs Markov models. The service based RAT selection algorithm has been studied using the proposed Markov model. Numerical results obtained from the proposed Markov model are presented.
\end{abstract}

\section{Introduction}

The future wireless network is expected to be a heterogeneous system, which integrates different Radio Access Technologies (RATs), such as GSM/EDGE Radio Access Network (GERAN), Universal Mobile Telecommunications System (UTRAN) and Wireless Local Area Network (WLAN), through a common platform. A major challenge arising is the Radio Resource Management (RRM) strategy. The common RRM (CRRM) strategy has been proposed in the literature to coordinate radio resource utilization across a number of RATs in an optimized way.

The RAT selection algorithm, including initial RAT selection and vertical handover (VHO), is one of the key research areas in CRRM. A suitable RAT selection algorithm can maximize system performance and the Quality of Service (QoS) by allocating users to the most suitable. Different RAT selection algorithms have been studied in the literature [1]. Service based RAT selection algorithm is studied in this paper. In the service based RAT selection algorithm, users will be allocated based on service types and network properties [2, 3].

The User Level Markov model can be used to analyze the probabilities of a single user being in different states. In the User Level Markov model, it is assumed that the network capacity is sufficient to serve the user, which means blocking and dropping will not happen. In [4], a simple User Level Markov model is presented by Falowo et al. This model is designed for an integrated UMTS/WLAN network, in which the UMTS has a wider coverage but higher service cost and the WLAN has a limited coverage but lower service cost. Falowo's model is relatively simple. In [5], a more complex User Level model is proposed by Hasib et al. In Falowo's model, if a user is in the hotspot area, where both UMTS and WLAN have coverage, the call will be allocated to WLAN only. However, in Hasib's model, a user in the hotspot area can be connected to WLAN, Wireless Wide Area Network (WWAN) or to both. This paper proposes a User Level Markov model for a three co-located RATs system based on an extension of the two co-located RATs Markov models.

The rest of the paper is organized as follows. Section 2 presents the proposed Markov model. In Section 3, the proposed model is applied to a simple scenario, where users only arrive and move within the hotspot area. In Section 4, the proposed model is applied to a relatively complex scenario, where users can arrive and move out of the hotspot area. Service based algorithm is studied in the two scenarios. Finally, this paper is concluded in Section 5.

\section{User Level Markov Model}

A user in the system can be in one of the following four states:

- State 0: Not connected

- State 1: Connected to GERAN

- State 2: Connected to UTRAN

- State 3: Connected to WLAN

Figure 1 shows the user state transition diagram. Let $\mathrm{P}_{0}, \mathrm{P}_{1}, \mathrm{P}_{2}$ and $\mathrm{P}_{3}$ be the probabilities of a user being in States $0,1,2$ and 3 respectively. The steady state probability transition matrix is:

$$
\mathrm{P}=\left[\begin{array}{llll}
P_{00} & P_{01} & P_{02} & P_{03} \\
P_{10} & P_{11} & P_{12} & P_{13} \\
P_{20} & P_{21} & P_{22} & P_{23} \\
P_{30} & P_{31} & P_{32} & P_{33}
\end{array}\right],
$$


where $0 \leq P_{i j} \leq 1$ for $\mathrm{i}, \mathrm{j}=0,1,2,3$;

The sum of each row of the matrix is 1 :

$$
\sum_{j=0}^{3} P_{i j}=1, \text { for } \mathrm{i}=0,1,2,3,
$$

$\mathrm{P}_{00}$ is the probability of no new user arriving. $\mathrm{P}_{01}, \mathrm{P}_{02}$ and $\mathrm{P}_{03}$ are the probabilities of a new arrival user being allocated to GERAN, UTRAN and WLAN respectively. $\mathrm{P}_{12}, \mathrm{P}_{13}, \mathrm{P}_{21}, \mathrm{P}_{23}, \mathrm{P}_{31}$ and $\mathrm{P}_{32}$ are the $\mathrm{VHO}$ probabilities. $\mathrm{P}_{11}, \mathrm{P}_{22}$ and $\mathrm{P}_{33}$ are the probabilities that an on going call is staying in the RAT currently serving it.

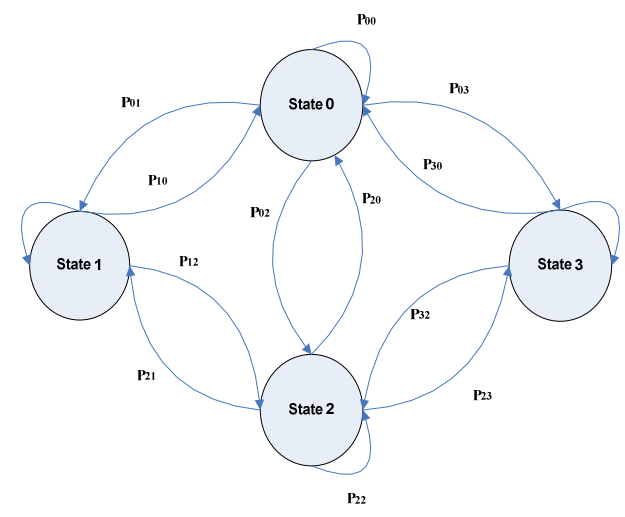

Figure 1. User state transition diagram

The steady state probabilities can be worked out by solving the following equation [6]:

$$
\pi=\pi \mathrm{P} \text {, }
$$

where $\pi$ is the state probability vector given by $\pi=$ $\left[\mathrm{P}_{0}, \mathrm{P}_{1}, \mathrm{P}_{2}, \mathrm{P}_{3}\right]$. Since a user can only be in the four states at any point of time,

$$
\mathrm{P}_{0}+\mathrm{P}_{1}+\mathrm{P}_{2}+\mathrm{P}_{3}=1 \text {. }
$$

In the next section, Markov models in different scenarios will be studied.

\section{Scenario 1}

In Scenario 1, it is assumed that users only arrive and move within the hotspot area. Two types of services, voice and data are considered. Voice users are allocated to GERAN, UTRAN and WLAN in order and data users are allocated in the inverse order [2]. A user is randomly determined as voice or data. We define the probabilities of a call to be voice and data are $P_{\text {voice }}$ and $P_{\text {data }}$ respectively.

$$
\mathrm{P}_{\text {voice }}+\mathrm{P}_{\text {data }}=1 \text {. }
$$

It is also assumed that new calls arrive according to a Poisson process with a mean arrival rate of $\lambda$. The call duration $\mathrm{T}_{\text {call }}$ is exponentially distributed with a mean of $1 / \mu$. The call completion rate is $\mu$. If we set the time unit of user state transition diagram to be the same as the call arrival and completion rate, the call completion probability is then to be:

$$
\mathrm{P}_{\text {term }}=\mu \text {. }
$$

The new call arriving probability is:

$$
\mathrm{P}_{\text {new }}=\lambda \text {. }
$$

The entire third row of the state transition matrix is 0 because a user will not be allocated to UTRAN when GERAN and WLAN have sufficient capacities.

It is assumed that call completion probabilities are irrelevant to the selected RAT:

$$
\mathrm{P}_{10}=\mathrm{P}_{30}=\mathrm{P}_{\text {term }} \text {. }
$$

The probability of a user staying in the idle state is:

$$
\mathrm{P}_{00}=1-\mathrm{P}_{\text {new }} \text {. }
$$

The probabilities of a new arrival user to be allocated into GERAN and WLAN are:

$$
\begin{aligned}
& \mathrm{P}_{01}=\mathrm{P}_{\text {new }} \times \mathrm{P}_{\text {voice }}, \\
& \mathrm{P}_{03}=\mathrm{P}_{\text {new }} \times \mathrm{P}_{\text {data }} .
\end{aligned}
$$

In service based algorithm, once the user is allocated to the most suitable RAT, it will not change to other RAT unless the current RAT can not support it any more. Therefore, the probabilities of an ongoing call to stay in GERAN and WLAN are

$$
\mathrm{P}_{11}=\mathrm{P}_{33}=\left(1-\mathrm{P}_{\text {term }}\right) \text {. }
$$

The other state transition probabilities are all 0 .

By using the above equations, the steady state probabilities can be worked out:

$$
\begin{gathered}
\mathrm{P}_{0}=\mathrm{P}_{\text {term }} /\left(\mathrm{P}_{\text {term }}+\mathrm{P}_{\text {new }}\right), \\
\mathrm{P}_{1}=\mathrm{P}_{\text {new }} \times \mathrm{P}_{\text {voice }} /\left(\mathrm{P}_{\text {term }}+\mathrm{P}_{\text {new }}\right), \\
\mathrm{P}_{2}=0, \\
\mathrm{P}_{3}=\mathrm{P}_{\text {new }} \times \mathrm{P}_{\text {data }} /\left(\mathrm{P}_{\text {term }}+\mathrm{P}_{\text {new }}\right) .
\end{gathered}
$$

Figure 2 shows the user state probabilities. It can be seen that the probability of a user to be served in GERAN is proportional to $\mathrm{P}_{\text {voice }}$ while the probability of a user to be served in WLAN is inverse proportional to $\mathrm{P}_{\text {voice. }}$ The probability of a user to be served in UTRAN is 0 . The probability of a user to be idle is constant because the user arrival rate and call duration are fixed.

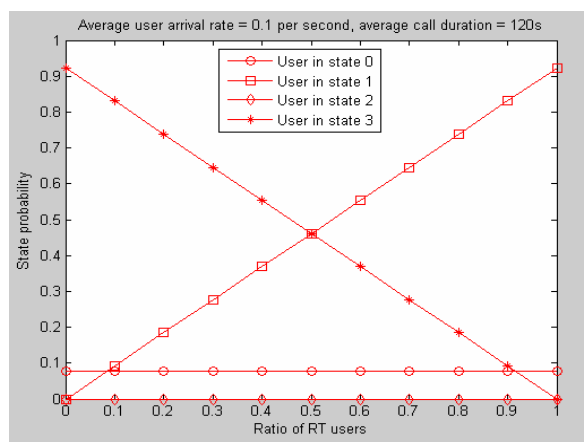

Figure 2. User state probabilities 


\section{Scenario 2}

In Scenario 2, it is considered that users can move within and outside the hotspot area. In this case, more states need to be defined:

- State 0: Not connected

- State 1: Within the hotspot area and connected to GERAN

- State 2: Within the hotspot area and connected to UTRAN

- State 3: Within the hotspot area and connected to WLAN

- State 4: Out of the hotspot area and connected to GERAN

- State 5: Out of the hotspot area and connected to UTRAN

Let $\mathrm{P}_{0}, \mathrm{P}_{1}, \mathrm{P}_{2}, \mathrm{P}_{3}, \mathrm{P}_{4}$ and $\mathrm{P}_{5}$ be the probabilities of a user being in State $0,1,2,3,4$ and 5 respectively.

$$
\mathrm{P}_{0}+\mathrm{P}_{1}+\mathrm{P}_{2}+\mathrm{P}_{3}+\mathrm{P}_{4}+\mathrm{P}_{5}=1 \text {. }
$$

The steady state probability matrix and user state transition diagram will be similar to the one before but in a larger scale.

The following definitions are made. The probability of a user residing in the hotspot area is $\mathrm{P}_{\text {in }}$. The probability of a user residing outside the hotspot area is then to be $P_{\text {out }}=1-\mathrm{P}_{\text {in }}$. The probability of a new call being voice call is $\mathrm{P}_{\text {voice }}$ and the probability of a new call being data call is $\mathrm{P}_{\text {data }}=1-\mathrm{P}_{\text {voice. }}$ The probability of a new call arrival in the hotspot area is $\mathrm{P}_{\text {new } \mathrm{h}}=\mathrm{P}_{\text {new }} \times \mathrm{P}_{\text {in }}$. The probability of a new call arrival outside the hotspot area is then to be $\mathrm{P}_{\text {new }} \times \mathrm{P}_{\text {out }}$. The probability of a user exiting the hotspot area during the session is $\mathrm{P}_{\mathrm{ex}}$. The probability of a user entering the hotspot area during the session is $\mathrm{P}_{\mathrm{en}}$.

We can use the same method to work out the steady state probabilities. The following default values are set:

\begin{tabular}{|l|l|}
\hline Call arrival rate & 0.1 per second \\
\hline Call duration & $120 \mathrm{~s}$ \\
\hline $\mathrm{P}_{\text {in }}$ & 0.5 \\
\hline $\mathrm{P}_{\text {voice }}$ & 0.5 \\
\hline
\end{tabular}

By assuming that the numbers of users moving into and out of the hotspot area is the same on average, we can get:

$$
\begin{gathered}
\mathrm{P}_{\mathrm{ex}} \times \mathrm{P}_{\text {in }}=\mathrm{P}_{\mathrm{en}} \times \mathrm{P}_{\text {out }}, \\
\text { If } \mathrm{P}_{\mathrm{in}}=1 \text { or } 0, \mathrm{P}_{\mathrm{ex}}=\mathrm{P}_{\mathrm{en}}=0 .
\end{gathered}
$$

As in Scenario 1, we will work out the elements in the matrix first. It is assumed that the call completion probabilities are the same in every RAT:

$$
\mathrm{P}_{10}=\mathrm{P}_{20}=\mathrm{P}_{30}=\mathrm{P}_{40}=\mathrm{P}_{50}=\mathrm{P}_{\text {term }} \text {. }
$$

The state transition probabilities for new calls are:

$$
\mathrm{P}_{00}=1-\mathrm{P}_{\text {new }} \text {, }
$$

$$
\begin{gathered}
\mathrm{P}_{01}=\mathrm{P}_{\text {new } \_\mathrm{h}} \times \mathrm{P}_{\text {voice, }}, \\
\mathrm{P}_{02}=0, \\
\mathrm{P}_{03}=\mathrm{P}_{\text {new }} \mathrm{h} \times \mathrm{P}_{\text {data }}, \\
\mathrm{P}_{04}=\mathrm{P}_{\text {new }} \times \mathrm{P}_{\text {out }} \times \mathrm{P}_{\text {voice }}, \\
\mathrm{P}_{05}=\mathrm{P}_{\text {new }} \times \mathrm{P}_{\text {out }} \times \mathrm{P}_{\text {data }} .
\end{gathered}
$$

Assuming that a call will initially connect to the RAT currently serving it,

$$
\mathrm{P}_{42}=\mathrm{P}_{43}=\mathrm{P}_{51}=\mathrm{P}_{53}=0 \text {. }
$$

The probabilities of a user moves into the hotspot area during the session are:

$$
\mathrm{P}_{41}=\mathrm{P}_{52}=\mathrm{P}_{\mathrm{en}} \times\left(1-\mathrm{P}_{\text {term }}\right) \text {. }
$$

The probabilities of a call moving out of the hotspot area and staying in the same RAT

$$
\mathrm{P}_{15}=\mathrm{P}_{24}=0 \text {. }
$$

The probabilities of a user in States 1, 2, 3 moving out of the hotspot area and staying in or VHO to GERAN during the session are:

$$
\begin{gathered}
\mathrm{P}_{14}=\mathrm{P}_{\mathrm{ex}} \times\left(1-\mathrm{P}_{\text {term }}\right), \\
\mathrm{P}_{34}=0 .
\end{gathered}
$$

The probabilities of a user in States 1, 2, 3 moves out of the hotspot area and stay in or VHO to UTRAN during the session are:

$$
\mathrm{P}_{25}=\mathrm{P}_{35}=\mathrm{P}_{\mathrm{ex}} \times\left(1-\mathrm{P}_{\text {term }}\right) \text {. }
$$

The probabilities of an ongoing call staying within the hotspot area are:

$$
\begin{gathered}
\mathrm{P}_{12}=\mathrm{P}_{13}=\mathrm{P}_{21}=\mathrm{P}_{22}=\mathrm{P}_{31}=\mathrm{P}_{32}=0, \\
\mathrm{P}_{23}=\left(1-\mathrm{P}_{\text {term }}\right) \times\left(1-\mathrm{P}_{\mathrm{ex}}\right) .
\end{gathered}
$$

The probabilities of an ongoing call staying outside the hotspot area are:

$$
\begin{gathered}
\mathrm{P}_{44}=\mathrm{P}_{55}=\left(1-\mathrm{P}_{\text {term }}\right) \times\left(1-\mathrm{P}_{\mathrm{en}}\right), \\
\mathrm{P}_{45}=\mathrm{P}_{54}=0 .
\end{gathered}
$$

By using the above equations, the steady state probabilities can be worked out:

$$
\begin{gathered}
\mathrm{P}_{0}=\mathrm{P}_{\text {term }} /\left(\mathrm{P}_{\text {term }}+\mathrm{P}_{\text {new }}\right) \\
\mathrm{P}_{1}=\mathrm{P}_{\text {in }}-\mathrm{P}_{2}-\mathrm{P}_{3}, \\
\mathrm{P}_{2}=\mathrm{d}_{3} \times \mathrm{P}_{5}, \\
\mathrm{P}_{4}=\left[\mathrm{c}_{1} \times \mathrm{P}_{\text {voice }} \times\left(\mathrm{P}_{0}+\mathrm{P}_{2} \times \mathrm{P}_{\text {data }} \times \mathrm{d}_{1} \times \mathrm{P}_{0} /\left(1-\mathrm{d}_{2}\right)\right] /\left[1-\mathrm{d}_{2} \times \mathrm{d}_{3} /\left(1-\mathrm{d}_{2}\right),\right.\right.
\end{gathered}
$$
$\left.\left.\mathrm{d}_{2}\right)-\mathrm{c}_{3}\right]$,

$$
\mathrm{P}_{5}=\mathrm{P}_{\text {out }}-\mathrm{P}_{4} \text {, }
$$

where $c_{1}=P_{\text {new }} \times P_{\text {out }}, c_{2}=P_{\text {ex }} \times\left(1-P_{\text {term }}\right)$, $\mathrm{c}_{3}=\left(1-\mathrm{P}_{\text {term }}\right) \times\left(1-\mathrm{P}_{\text {en }}\right), \mathrm{d}_{1}=\mathrm{P}_{\text {new } \mathrm{h}} \times \mathrm{P}_{\text {voice }}$

$$
\begin{gathered}
\mathrm{d}_{2}=\left(1-\mathrm{P}_{\text {term }}\right) \times\left(1-\mathrm{P}_{\text {ex }}\right), \mathrm{d}_{3}=\mathrm{P}_{\text {en }} \times\left(1-\mathrm{P}_{\text {term }}\right) . \\
\mathrm{P}_{\text {in }}=\mathrm{P}_{1}+\mathrm{P}_{2}+\mathrm{P}_{3}=1-\mathrm{P}_{0}-\mathrm{P}_{\text {out }}, \\
\mathrm{P}_{\text {out }}=\mathrm{P}_{4}+\mathrm{P}_{5}=\left(\mathrm{c}_{1} \times \mathrm{P}_{0}+\mathrm{c}_{2} \times\left(1-\mathrm{P}_{0}\right)\right) /\left(1+\mathrm{c}_{2}-\mathrm{c}_{3}\right) .
\end{gathered}
$$

Figure 3 and 4 show that with the increase of new user arrival rate and/or call duration, the probability of a user being idle decreases and the probability of a user being served increases.

Figure 5 shows the probabilities of a user being in different states with the increase of the probability of new users arriving in the hotspot area. $\mathrm{P}_{2}$ depends on the number of data users served in UTRAN out of the 
hotspot area and the probability of users cross the border. Higher number of users out of the hotspot area will cause lower probability of users entering the hotspot area. The maximum value of $\mathrm{P}_{2}$ occurs at $\mathrm{P}_{\mathrm{h}}=0.5$.

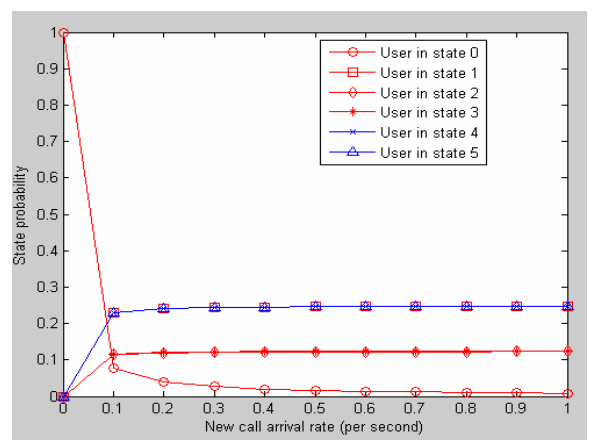

Figure 3. User state probabilities with the increase of the probability of new user arrival rate

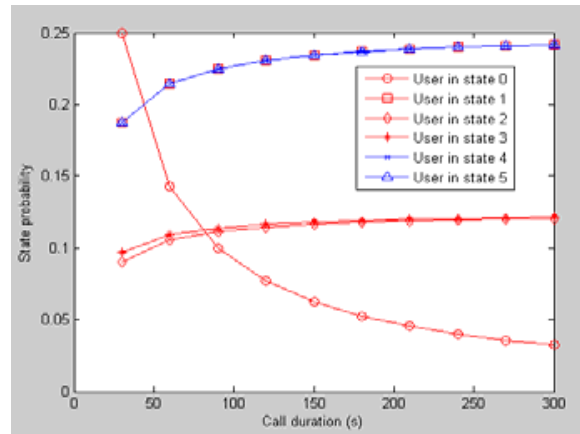

Figure 4. User state probabilities with the increase of call duration

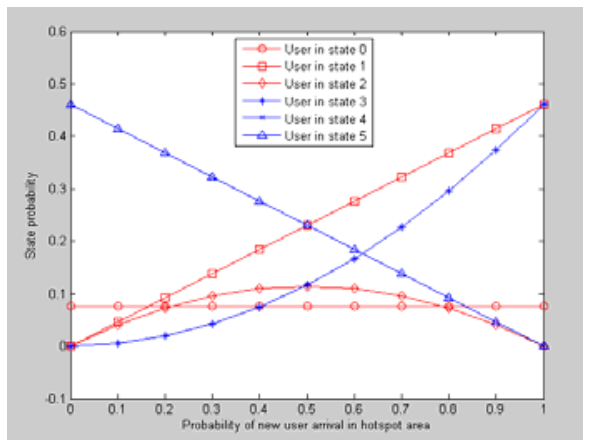

Figure 5. User state probabilities with the increase of the probability of new user arrival in the hotspot area

Figure 6 shows that the probabilities of a user being in different states with the increase of the probability of a user being a real time (RT) user. The probabilities of a user to be served in GERAN/UTRAN increases while the probabilities of the user to be served in WLAN decreases.

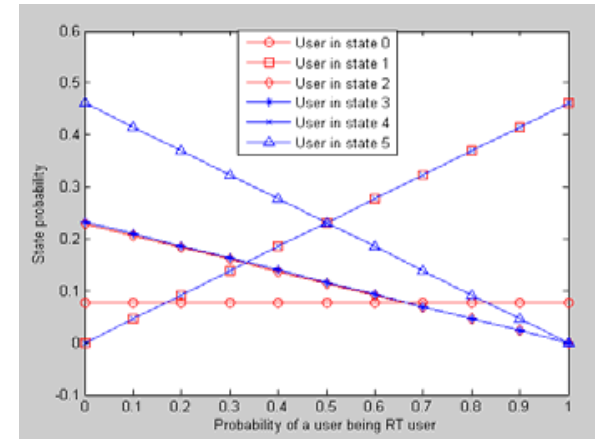

Figure 6. User state probabilities of a user being in different states with the increase of the probability of a user being a real time (RT) user

\section{Conclusions}

This paper proposes a User Level Markov model for a three overlapped RATs network (GERAN/UTRAN/WLAN). Service based RAT selection algorithm has been studied using the proposed Markov model. Two scenarios, user can and cannot move out of the hotspot area have been considered. The numerical results show the user state probability patterns under different conditions.

\section{Acknowledgment}

This work is partly sponsored by the Commonwealth Scientific and Industrial Research Organisation (CSIRO) Information and Communication Technologies (ICT) centre.

\section{References}

[1] L. Wu and K. Sandrasegaran, "A Survey on Common Radio Resource Management," in The Second Australia Conference on Wireless Broadband and Ultra Wideband Communications (Auswireless07), Sydney, Australia, 2007, p. 66.

[2] A. Baraev, L. Jorguseski, and R. Litjens, "Performance Evaluation of Radio Access Selection Procedures in MultiRadio Access Systems," in Proceedings of WPMC '05, Aalborg, Denmark, 2005.

[3] J. Pérez-Romero, O. Sallent, and R. Agustí, "Policy-based Initial RAT Selection algorithms in Heterogeneous Networks," in 7th Mobile Wireless Communication Networks (MWCN), Marrakech, Morocco, 2005, pp. 1-5.

[4] O. E. Falowo and H. A. Chan, "Joint Call Admission Control for Next Generation Wireless Network," in Canadian Conference on Electrical and Computer Engineering, 2006 (CCECE '06), Ottawa, Ont, 2006, pp. 1151-1154.

[5] A. Hasib and A. O. Fapojuwo, "Analysis of Common Radio Resource Management Scheme for End-to-End QoS Support in Multiservice Heterogeneous Wireless Networks," IEEE Transactions on Vehicular Technology, vol. 57, pp. 24262439, July 2008.

[6] S. M. Ross, Introduction to probability models, Third ed. Orlando: Academic Press, 1985. 